\title{
Classificação visual e mecânica da espécie Cryptomeria japonica D. Don para utilização em madeira laminada colada
}

\author{
Visual and mechanical classification of the specie Cryptomeria japonica D. Don \\ for using as glued laminated timber
}

\begin{abstract}
Talitha Olivera Rosa ${ }^{\mathrm{I}}$, Helena Cristina Vieira ${ }^{\mathrm{I}}$, Rodrigo Figueiredo Terezo ${ }^{\mathrm{II}}$, Alexsandro Bayestorff da Cunha' ${ }^{\mathrm{II}}$, Carlos Augusto de Paiva Sampaio"II, Charline Zangali ${ }^{\mathrm{IV}}$, Gabriel Oliveira Rosa ${ }^{\mathrm{v}}$, Deyvis Borges Waltrick ${ }^{\mathrm{IV}}$
\end{abstract}

\begin{abstract}
Resumo
O objetivo foi avaliar as propriedades mecânicas da madeira, a influência da classificação visual de lâminas de Cryptomeria japonica D. Don no desempenho mecânico para uso em elementos estruturais em MLC, comparando com os requisitos mínimos exigidos pelas normas brasileira ABNT NBR 7190 (1997) e europeia EN 338 (2016). Os ensaios para caracterização física e mecânica da madeira sólida seguiram as recomendações das normas COPANT 461 (1972), 464 (1972) e 555 (1973) para densidade básica, resistência à compressão paralela às fibras e resistência à flexão estática, respectivamente; e ABNT NBR 7190 (1997) para resistência ao cisalhamento. As 80 lâminas de madeira foram produzidas com dimensões de 6,0 x 1,5 x 220,0 cm (largura x espessura x comprimento) e posteriormente classificadas visualmente conforme a norma americana ASTM D245 (2006) e pelo módulo de elasticidade - $\mathrm{E}_{\mathrm{w}}$ (ASTM D4761 (2013)). Ao todo, 20 vigas de MLC foram confeccionadas, sendo estas formadas pela combinação das lâminas classificadas nas classes visuais: Testemunha (lâminas limpas, ou seja, sem defeitos), T1 (limpas e estrutural especial - SE), T2 (S1, S2, S3) e T3 (limpas, SE, S1, S2, S3), as vigas foram avaliadas em testes mecânicos de resistência à flexão estática e resistências à tração normal às fibras e cisalhamento para linha de cola. Os resultados indicaram que como madeira sólida a espécie não apresentou resistência mínima para que seja classificada como C-20 pela ABNT NBR 7190 (1997), entretanto a espécie foi classificada como classe estrutural C-16 pela norma EN 338. As vigas de MLC apresentaram valores dentro do mínimo exigido para a classe C-20, conforme a ABNT NBR 7190 (1997). A utilização de lâminas com nós classificados visualmente não diminuiu a resistência das vigas de MLC quando comparado com vigas produzidas com lâminas sem nós.
\end{abstract}

Palavras-chaves: Elasticidade; Uso estrutural; Classificação visual

\footnotetext{
IEngenheira Florestal, MSc., Doutoranda Departamento de Engenharia Florestal, Centro Jardim Botânico, Universidade Federal do Paraná, Av. Prefeito Lothário Meissner, 632, CEP 80210-170, Curitiba (PR), Brasil. rosa.talitha@gmail.com (ORCID: 0000-0002-3639-2240) / lena.cristin@ hotmail.com (ORCID: 0000-0002-3639-2240)

II Engenheiro Civil / Engenheiro Florestal, Dr., Professor do Departamento de Engenharia Florestal, Centro de Ciências Agroveterinárias, Universidade do Estado de Santa Catarina, Av. Luiz de Camões, 2090, CEP 88520-000, Lages (SC), Brasil. rodrigo.terezo@udesc.br (ORCID: 00000002-7562-1931) / alexsandro.cunha@udesc.br (ORCID: 0000-0001-5554-5276)

III Engenheiro Agrícola, Dr., Professor do Departamento de Agronomia, Centro de Ciências Agroveterinárias, Universidade do Estado de Santa Catarina, Av. Luiz de Camões, 2090, CEP 88520-000, Lages (SC), Brasil. carlos.sampaio@udesc.br (ORCID: 0000-0003-3931-3840)

IV Engenheiro(a) Florestal, Mestrando(a) do Departamento de Engenharia Florestal, Centro de Ciências Agroveterinárias, Universidade do Estado de Santa Catarina, Av. Luiz de Camões, 2090, CEP 88520-000, Lages (SC), Brasil. eng.borges@outlook.com (ORCID: 0000-0002-1004-1370) / charlineeng@gmail.com (ORCID: 0000-0002-7667-3389)

Estudante do Curso de Engenheira Florestal, Departamento de Engenharia Florestal, Centro de Ciências Agroveterinárias, Universidade do Estado de Santa Catarina, Av. Luiz de Camões, 2090, CEP 88520-000, Lages (SC), Brasil. gabrielrosa.or@hotmail.com (ORCID: 0000-0002-9424-1256)
} 


\begin{abstract}
The objective was to evaluate the mechanical properties, the visual classification influence of Cryptomeria japonica D. Don at the mechanical performance to use it as glulam structural elements, comparing with the minimum requirements demanded by the Brazilian Standard ABNT NBR 7190 (1997) and European Standard EN 338 (2006). The tests for the characterization of the physical and mechanical properties of solid timber followed the recommendations of COPANT 461 (1972), 464 (1972), 555 (973) for density of timber, parallel compression to grain and bending, respectively; and ABNT NBR 7190 (1997) for the shear strength. The classification of the 80 laminate of timber with dimension of $6.0 \times 1.5 \times 220.0 \mathrm{~cm}$ were made by visual classification according to the American Standard ASTM D245 (2006) and by the modulus of elasticity - MOE (ASTM D4761 (2013)). Altogether, 20 beams of GLULAM were made, these formed by the combination of the laminated classified in the visual grades: Control (clear laminate), T1 (clear and special structural - SE), T2 (S1, S2, S3) and T3 (clear, SE, S1, S2, S3), the beams were evaluated by bending, tension perpendicular to grain and shear strength in the glued line. The results indicated the species as solid wood does not have the minimum resistance to be classified as C-20 by the ABNT NBR 7190 (1997), but the species was classified as structural grade in the C-16 by the EN 338 Standard. The GLULAM beams showed the minimum demanded values to the C-20 structural class according to the ABNT NBR 7190 (1997). The using of laminated with visually classified knots did not decrease the glulam beams strength when comparing to the beams made with laminated without knots.
\end{abstract}

Keywords: Elasticity;Structural purpose; Visual grade

\title{
Introdução
}

A madeira é um material renovável com excelente capacidade de carregamento em relação a sua própria massa. Assim, pode ser utilizada de maneira estrutural na forma de madeira laminada colada (MLC).

A MLC é um material compósito formado a partir da colagem, de topo e adjacente, de lâminas de madeira selecionadas e dispostas com as fibras paralelas entre si. Como a MLC é um produto pouco utilizado no Brasil, pesquisas visam compreender melhor as características desse compósito utilizando diferentes espécies florestais.

Terezo e Szücs (2010) determinaram o potencial do paricá (Schizolobium amazonicum) para a produção de MLC. Os resultados desses autores para as resistências das vigas de MLC de paricá, cuja densidade da madeira é de $0,49 \mathrm{~g} . \mathrm{cm}^{-3}$, propiciaram a classificação como C-20 para uso estrutural, conforme a ABNT NBR 7190 (1997). Cunha e Matos (2010) classificaram visualmente a madeira de Pinus taeda, utilizada para a produção de MLC, aliada à classificação mecânica do módulo de elasticidade longitudinal obtido com Stress Wave. Os autores concluíram que somente a classificação visual não resulta em vigas de MLC com alta resistência, sendo necessário aplicar a classificação visual e mecânica, produzindo vigas com maior rigidez. Ambos os trabalhos sugerem que madeiras de baixa densidade podem apresentar resistências mínimas para uso estrutural, se as metodologias para classificação dessas madeiras forem aplicadas.

O produto estrutural MLC tem como sua composição básica a madeira, tornando-o propício à heterogeneidade, devido à ocorrência de nós e de outros defeitos naturais inerentes a estrutura do tronco das árvores e das etapas de desdobro, secagem e armazenamento da madeira. Assim, o setor produtivo precisa reconhecer a importância das normas de qualidade da madeira, para suprir e manter os produtos com desempenho adequado, com o menor desperdício possível (MOORE; COWN, 2017).

A classificação mecânica ou visual é uma das formas de se avaliar a qualidade da MLC, bem como a implantação de testes físicos e mecânicos em determinados lotes da madeira 
(ALMEIDA et al., 2013).

A classificação mecânica da elasticidade das lâminas utilizadas na confecção do MLC, segundo Bodig e Jayne (1993), também incrementa o desempenho da MLC e possibilita um controle da capacidade de carga do material. Segundo os autores, as lâminas de maior elasticidade devem ser posicionadas nas zonas de tração e compressão e as de menor elasticidade devem ser posicionadas nas zonas centrais, aumentando, assim, a resistência do conjunto MLC.

Para determinar o uso estrutural de lâminas de madeira é utilizada a classificação visual, que consiste na inspeção das 6 superfícies: bordas, laterais e extremidades de cada peça. A norma americana ASTM D 245 (2006) prevê a classificação visual da madeira serrada de coníferas, a qual determina quatro classes de qualidade visual de acordo com a proporção que o nó ocupa em relação à área da seção transversal da peça.

Como os nós são os defeitos mais comumente encontrados em madeira de coníferas, torna-se um importante parâmetro para a classificação visual. Contudo, em uma indústria de MLC que possua um processo de classificação controlado, apenas as áreas da madeira consideradas críticas, com nós que ultrapassem o máximo aceito pelas normas, são eliminadas e todo o material remanescente pode ser utilizado (LUKACEVIC; FÜSSL, 2014).

No Brasil, o mercado de MLC é composto principalmente por lâminas dos gêneros Eucalyptus e Pinus, no entanto, frente à crescente demanda, buscam-se espécies alternativas, como a Cryptomeria japonica D. Don. Essa espécie é uma conífera originária do Japão e no Brasil é conhecida como cedro japonês ou pinheiro-japonês. A espécie foi introduzida como alternativa aos plantios de Pinus spp. nos estados de São Paulo, sul de Minas Gerais, Paraná e Santa Catarina (CARPANEZZI et al., 1988).

Essa espécie apresenta características como fuste reto, rápido crescimento, alta durabilidade da madeira e facilidade de processamento (IWAKIRI et al., 2015), tornando viável estudos que verifiquem o potencial da espécie para o uso em construções.

Desse modo, este trabalho objetivou avaliar as propriedades mecânicas da madeira, bem como a influência da classificação visual de lâminas de Cryptomeria japonica no desempenho mecânico para uso em elementos estruturais em MLC, conforme as exigências normativas brasileira e europeia.

\section{Material e método}

Este trabalho foi realizado com árvores da espécie Cryptomeria japonica D. Don obtidas de um povoamento localizado no município de São José do Cerrito, estado de Santa Catarina. A região tem altitude média de 879 m, latitude de $27^{\circ} 39^{\prime} 39 ; 47^{\prime}$ 'S e longitude 50 34'39,48’'W. O clima da região, segundo a classificação de Köppen, é do tipo $\mathrm{Cfb}$, apresentando uma precipitação média anual de $1.300 \mathrm{~mm}$.

Desse povoamento foram selecionadas aleatoriamente 30 árvores com 13 anos de idade. Após o seccionamento das árvores em toras, com comprimento médio de $220,0 \mathrm{~cm}$ e diâmetro médio de 23,5 cm, confeccionou-se 80 lâminas com dimensões médias de $6,0 \mathrm{~cm}$ x 1,5 cm x 220,0 $\mathrm{cm}$ (largura x espessura x comprimento, respectivamente). Das 30 árvores citadas anteriormente, algumas toras foram desdobradas para preparação dos corpos de prova de caracterização das propriedades físicas e mecânicas da espécie.

Na Figura 1, observam-se, de maneira esquemática, os procedimentos metodológicos utilizados. 


\section{Figura 1 - Organograma da metodologia empregada, desde o corte das árvores até a caracterização tecnológica das vigas de Madeira Laminada Colada}

Figure 1 - Organogram of the methodology used, for tree cutting to the characterization of the glulam beams

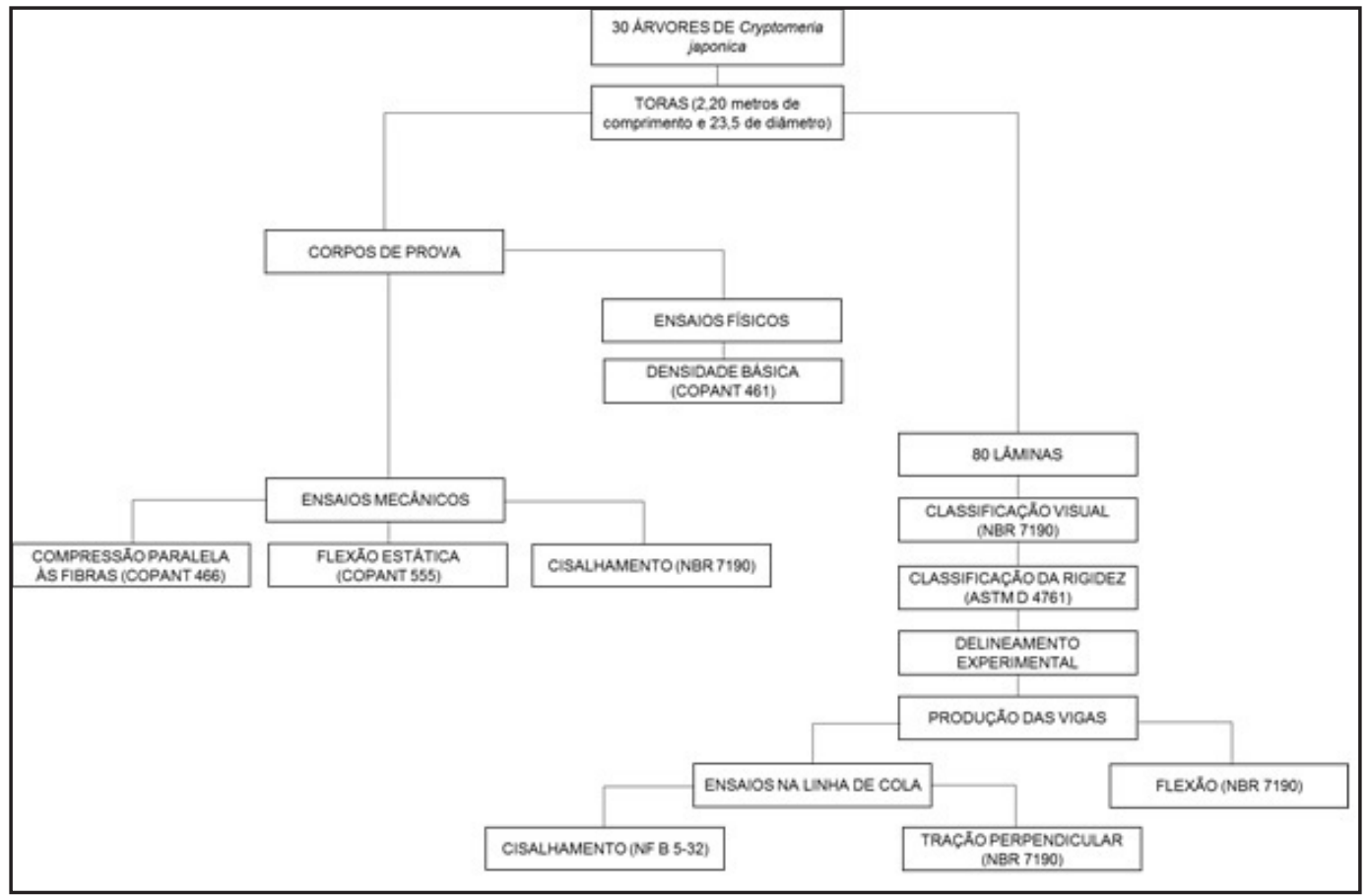

Fonte: Autores (2019)

\section{Caracterização física e mecânica da madeira}

A caracterização da resistência foi realizada empregando-se 15 corpos de prova para cada ensaio mecânico. Determinou-se a densidade básica pela COPANT 461 (1972), compressão paralela às fibras pela COPANT 464 (1972), flexão estática pela COPANT 555(1973), e cisalhamento pela ABNT NBR 7190 (1997).

\section{Classificação visual e da rigidez das lâminas}

Para a determinação das classes visuais das lâminas, os nós da madeira foram medidos nas faces radial e tangencial e classificados conforme a proporção de área que ocupam na seção transversal da peça. Conforme as premissas da ASTM D 245 (2006), a proporção da área que os nós ocupam na secção transversal da peça foram utilizadas para determinar as classes de classificação visual da madeira, ou seja, Classe Estrutural Especial (SE), Classe no 1 (S1), Classe no 2 (S2) e Classe nº 3 (S3). As proporções das áreas admissíveis para os nós foram obtidas com base nas equações para a limitação dos nós e nas razões de resistência à flexão descritas no apêndice da norma ASTM D 245 (2006).

Como observado por Carreira, Dias e Calil Junior. (2004), as proporções dentro das classes são praticamente constantes, e pode-se limitar em função da posição e proporção da área da seção transversal que o nó ocupa da peça. As proporções dos limites aceitos em cada classe são apresentadas na Tabela 1 . 
Tabela 1 - Limite de área que o nó ocupa em relação à seção transversal da peça de madeira

Table 1 - Limit of area that the knot occupies in relation to the cross section of the wood piece

\begin{tabular}{lcccc}
\hline Posição dos nós & SE & S1 & S2 & S3 \\
\hline Face e canto do lado & $20 \%$ & $25 \%$ & $33 \%$ & $50 \%$ \\
Centro da face & $35 \%$ & $45 \%$ & $50 \%$ & $75 \%$ \\
\hline
\end{tabular}

Fonte: Autores (2019)

Desse modo, as lâminas foram classificadas em cada uma das classes SE, S1, S2, S3 e as lâminas que não apresentaram nós, foram definidas como Testemunhas.

A elasticidade das lâminas foi obtida por ensaio não destrutivo de resistência à flexão a 3 pontos, de acordo com a norma ASTM D 4761 (2013). Nesta, as lâminas foram posicionadas no sentido de menor inércia, bi apoiadas, com um vão de $1,90 \mathrm{~m}$, aplicando-se um peso de $2 \mathrm{kgf}$ no seu centro, mantendo todas as peças com a força em uma zona de deformação elástica. A leitura do deslocamento da linha neutra foi feita com o auxílio de uma régua graduada posicionada no centro do vão (Figura 2).

Figura 2 - Metodologia para determinar o módulo de elasticidade $\left(E_{\mathrm{w}}\right)$ de lâminas de madeira de Cryptomeria japonica. (a) Leitura na posição inicial da lâmina sem a aplicação do peso. (b) leitura da deformação após aplicação do peso. (c) Exemplo do deslocamento da lâmina de madeira com a aplicação do peso no ponto central do vão

Figure 2 - Methodology to measure the Modulus of Elasticity of Cryptomeria japonica laminated. (a) Reading in the initial position of the laminated timber without the application of the weight. (b) reading of the deformation after application of the weight. (c) Example of the displacement of the timber laminated by applying the weight to the center point of the gap

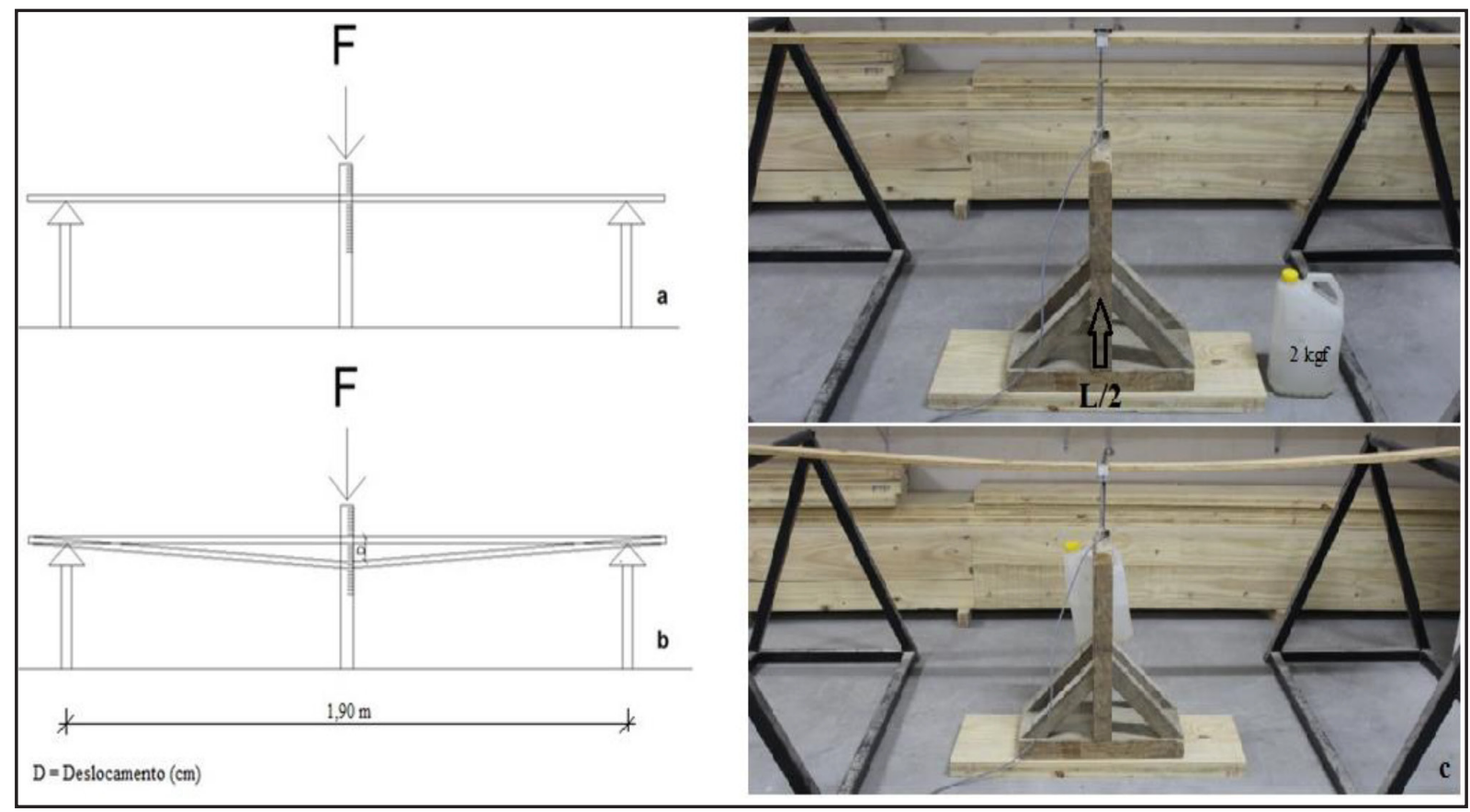

Fonte: Autores (2019) 
Em seguida, mediu-se na régua o ponto inicial correspondente à altura da peça sem emprego da força e aplicou-se o peso no ponto central fazendo a leitura da posição da peça (posição final) na régua. Utilizando a diferença entre a posição final (com força aplicada) e a inicial (sem força aplicada), obteve-se a deformação ou deslocamento (D) da linha neutra. Posteriormente repetiu-se o processo para o verso da lâmina, obtendo-se uma segunda leitura. $\mathrm{O}$ deslocamento final da peça foi determinado pela média.

O cálculo do módulo de elasticidade $\left(\mathrm{E}_{\mathrm{w}}\right)$ foi obtido por:

$$
E_{W}=\frac{F L^{3}}{48 D l^{4}}
$$

Equação 1.

Em que: $\mathrm{E}_{\mathrm{w}}=$ módulo de elasticidade em $\mathrm{MPa} ; \mathrm{F}$ = força aplicada no centro do vão em $\mathrm{kgf} ; \mathrm{L}$ = vão entre apoios em $\mathrm{mm}$; $\mathrm{D}$ = deslocamento médio da peça em $\mathrm{mm}$; I = momento de inércia da peça em $\mathrm{mm}^{4}$.

\section{Confecção e ensaio das vigas de MLC}

Os tratamentos foram delineados da seguinte forma: tratamento Testemunha foi composto por lâminas limpas; tratamento T1 foi composto por lâminas limpas e da classe SE; tratamento T2 foi composto por lâminas S1; S2; S3 e o tratamento T3 que foi composto com lâminas de todas as classes especificadas pela norma de classificação visual (Figura 3).

\section{Figura 3 - Representação dos conjuntos confeccionados para diferenciar os tratamentos utilizados para a produção das vigas de Madeira Laminada Colada}

Figure 3 -Representation of the sets made to differentiate the treatments used for the production of the glulam beams

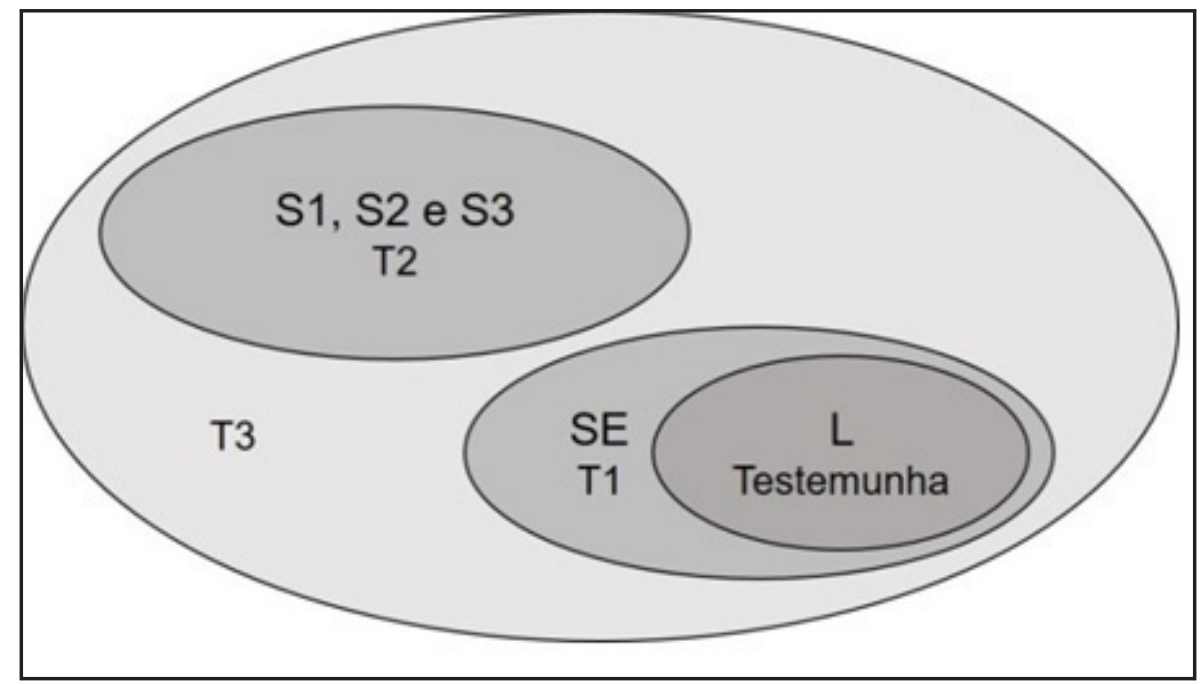

Fonte: Autores (2019)

Em seguida, procedeu-se a disposição das lâminas para a confecção das vigas de acordo com o $E_{\mathrm{w}}$, priorizando-se as de maior rigidez nas periferias e as de menor rigidez no centro. Conforme observado por Bodig e Jayne (1993), essa disposição sistemática das lâminas no produto MLC aumenta a rigidez do compósito. Para cada tratamento foram confeccionadas 5 vigas, com 4 lâminas na composição, que foram aplainadas e posteriormente limpas com um jato de ar para evitar resíduos no processo de colagem. 
O adesivo utilizado para a união das lâminas foi a base de poliuretano, com tempo de abertura de 20 min e gramatura de $200 \mathrm{~g} . \mathrm{cm}^{-2}$, sendo que sua quantidade foi controlada com o auxílio de uma balança digital. Em seguida, as vigas foram contidas lateralmente para evitar deslizamento das lâminas e prensadas com 1,0 MPa durante 6 horas. Após repouso de 3 dias para o término do tempo de cura da cola, as vigas foram aplainadas para retirada do excesso de adesivo e separadas em corpos de prova para o ensaio de resistência à flexão estática, conforme ABNT NBR 7190 (1997) e para avaliação da linha de cola, pelos testes de resistências à tração normal às fibras, conforme ABNT NBR 7190 (1997) e cisalhamento da linha de cola, conforme AFN NF B 5-32 (1942). Os corpos de prova para ambos os ensaios (resistência à flexão estática e avaliação da linha de cola) foram retirados de vigas não ensaiadas, totalizando 12 amostras para cada ensaio.

A avaliação estatística das variáveis foi realizada pelos testes de normalidade (KolmogorovSmirnov), variância (Bartlett) e a comparação de médias foi realizada pelo teste de Scott-Knott, a $5 \%$ de significância.

\section{Resultados e discussão}

\section{Caracterização mecânica e densidade básica}

A densidade básica da madeira de Cryptomeria japonica usada neste trabalho foi de 266 kg.m ${ }^{-3}$, similar ao encontrado por Carneiro, Bitencourt, Muniz, (2009), de $255 \mathrm{~kg} \cdot \mathrm{m}^{-3}$ e inferior ao valor de $433 \mathrm{~kg} \cdot \mathrm{m}^{-3}$ obtido por Fonte et al. (2017). As diferenças de $4,13 \%$ e $-62 \%$, respectivamente, dos valores observados neste trabalho com os autores citados podem estar relacionadas à idade das árvores, solo, condições climáticas, dentre outros. As árvores do estudo de Carneiro, Bitencourt, Muniz, (2009) possuíam 14 anos, provavelmente, sem formação de lenho adulto, e o sítio plantado está localizando na latitude $27^{\circ}$ e longitude $50^{\circ}$, sítio próximo ao deste trabalho. A diferença de $-62 \%$ com o trabalho de Fonte et al. (2017) pode ser devido às idades das árvores, já que, no segundo trabalho, as mesmas tinham 35 anos de idade, e esta poderia apresentar lenho adulto já formado. Contudo, a madeira da Cryptomeria japonica é considerada uma madeira de baixa densidade.

Os resultados médios da resistência e módulo de elasticidade à compressão paralela às fibras, resistência e módulo de elasticidade à flexão estática, resistência ao cisalhamento e resistência à tração paralela às fibras da madeira são apresentados na Tabela 2.

Tabela 2 - Valores médios das propriedades mecânicas da madeira de Cryptomeria japonica

Table 2 - Mean values of the mechanical properties of Cryptomeria japonica

\begin{tabular}{lccccccc}
\hline & \multicolumn{2}{c}{$\begin{array}{c}\text { Compressão paralela } \\
(\mathbf{M p a})\end{array}$} & \multicolumn{2}{c}{$\begin{array}{c}\text { Flexão } \\
(\mathbf{M P a})\end{array}$} & $\begin{array}{c}\text { Tração paralela } \\
(\mathbf{M P a})\end{array}$ & $\begin{array}{c}\text { Cisalhamento } \\
(\mathbf{M P a})\end{array}$ \\
\hline & $\mathbf{f}_{\mathrm{c}, 0}$ & $\mathbf{E w}$ & $\mathbf{f}_{\mathrm{m}}$ & $\mathbf{E w}$ & $\mathbf{f}_{\mathrm{t}, 0}$ & $\mathbf{f}_{\mathbf{v}}$ \\
\hline Característica & 16,55 & - & 17,44 & - & 30,62 & 5,37 \\
Média & 17,61 & 863,07 & 27,06 & $4.427,29$ & 38,34 & 6,22 \\
$\mathbf{C V}(\%)$ & $9,05 \%$ & $27,76 \%$ & $23,43 \%$ & $38,56 \%$ & $24,35 \%$ & $14,90 \%$ \\
\hline
\end{tabular}

Fonte: Autores (2019)

Em que: D.P. = Desvio padrão; CV $(\%)=$ Coeficiente de variação em porcentagem. $\mathrm{f}=$ resistência e Ew $=$ Módulo de elasticidade. 
Os resultados mostram que a madeira de Cryptomeria japonica com 13 anos de idade não apresenta os valores mínimos especificados pela ABNT NBR 7190 (1997) para ser considerada como madeira de uso estrutural na classe $\mathrm{C}-20$, pois a resistência característica mínima à compressão é de $20 \mathrm{MPa}$ e a densidade básica mínima de $400 \mathrm{~kg} \cdot \mathrm{m}^{-3}$. Já pela EN 338 (2016), que avalia a classificação da madeira para uso estrutural pelo ensaio de flexão estática, a espécie atenderia os requisitos da classificação C-16.

Os valores médios das propriedades mecânicas também foram próximos aos de Carneiro, Bitencourt, Muniz (2009), indicando que a espécie com idade de 13 anos apresenta classes de resistência inferiores. As baixas propriedades mecânicas podem estar associadas à ocorrência de lenho juvenil, uma vez que a proporção deste é maior em árvores provenientes de reflorestamentos colhidas em idades mais precoces, influenciando diretamente nas propriedades mecânicas (VIDAURRE et al., 2011). Os altos coeficientes de variação (CV) corroboram que existe variabilidade dentro da árvore na extração dos corpos de prova no sentido medula-casca.

Na Figura 4, está apresentado uma exemplificação da quantidade de nós permitidos em cada classe estrutural para a espécie Cryptomeria japonica. Observa-se que embora os nós sejam mais frequentes ao passar para classes estruturais mais baixas, estes apresentam diâmetro pequenos. Assim, mesmo que exista uma quantidade aparentemente grande de nós dentro de $15 \mathrm{~cm}$ de comprimento, conforme especificado pela ASTM D245 (2006), a somatória destes não descarta a lâmina de ser usada na classe S3.

\section{Figura 4 - Distribuição de lâminas de Cryptomeria japonica com nós classificados visualmente pela norma ASTM D245 (2006)}

Figure 4 - Distribution of Cryptomeria japonica laminated with knots visually classified by the ASTM D245 (2006) standard

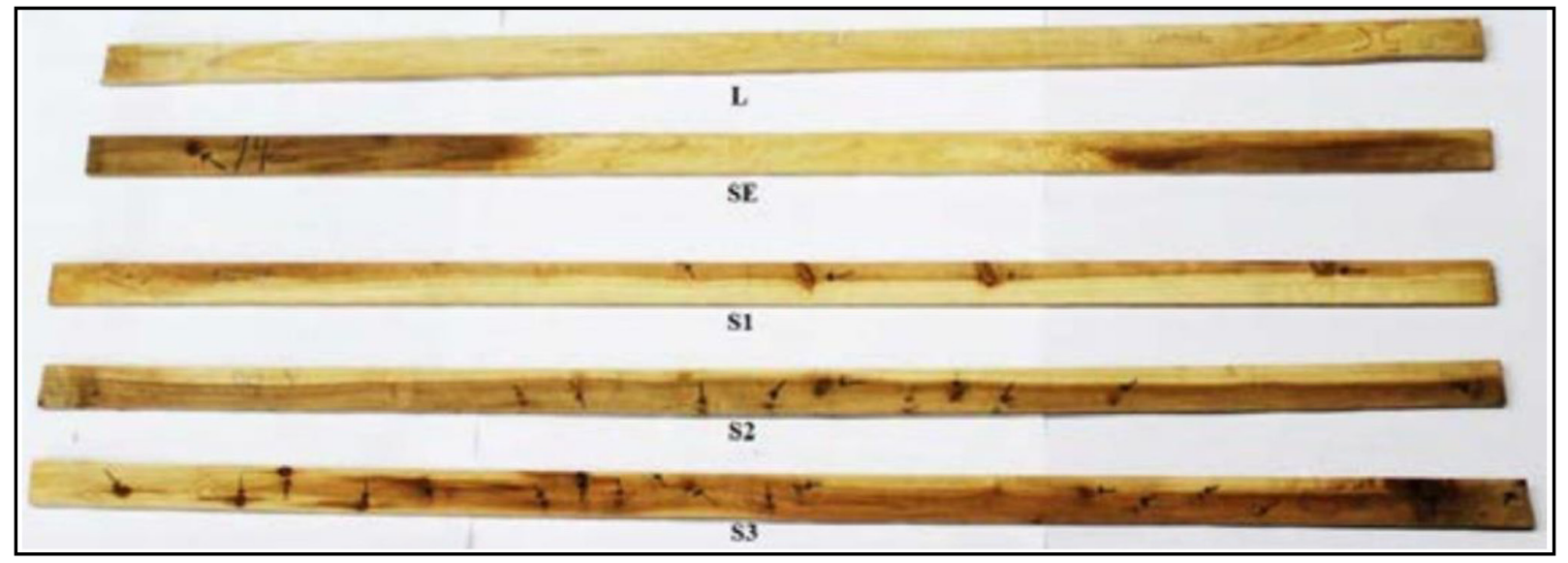

Fonte: Autores (2019)

$\mathrm{Na}$ Tabela 3, apresentam-se os resultados obtidos de resistência à tensão normal às fibras, resistência ao cisalhamento e o módulo de elasticidade à flexão estática para os diferentes tratamentos.

Não foram constatadas diferenças estatísticas entre os tratamentos para a tensão de ruptura axial e cisalhamento ( $\mathrm{p}$-valor 0,0851 ) e módulo de elasticidade (p-valor 0,3488), nem aumento ou redução das forças características entre os tratamentos.

Ao comparar os valores de resistência axial característicos dos tratamentos com os valores testemunha, nota-se que os tratamentos $\mathrm{T} 1$ e $\mathrm{T} 2$ apresentaram resistências de $2,53 \%$ e $0,34 \%$ menores que o testemunha e o T3 apresenta uma resistência de 5,91\% maior em relação à testemunha. Assim, apenas o uso de lâminas L e SE (T1) não garante que a resistência seja maior 
do que ao usar lâminas com classificação S1, S2 e S3 (T2), já que a própria heterogeneidade da madeira pode influenciar na resistência.

\section{Tabela 3 - Comparação estatística das tensões e MOE entre os tratamentos de Cryptomeria japonica}

Table 3 - Statistical comparison of tensions and modulus of elasticity among the treatments of Cryptomeria japonica

\begin{tabular}{|c|c|c|c|}
\hline \multirow{2}{*}{ Tratamento } & \multicolumn{2}{|c|}{ Resistência à flexão (MPa) } & \multirow{2}{*}{$\begin{array}{c}\text { Módulo de } \\
\text { Elasticidade (MPa) }\end{array}$} \\
\hline & Axial & Cisalhamento & \\
\hline Testemunha & $51,04 \mathrm{a}$ & $1,22 \mathrm{a}$ & $9.164,16 \mathrm{a}$ \\
\hline (CV\%) & 12,21 & 12,21 & 28,24 \\
\hline fm.k (12\%) & 47 & & \\
\hline fvo,k (12\%) & 1,1 & & \\
\hline T1 & $50,30 \mathrm{a}$ & $1,20 \mathrm{a}$ & $9.084,19 \mathrm{a}$ \\
\hline (CV\%) & 7,72 & 7,72 & 20,2 \\
\hline fm.k $(12 \%)$ & 48,19 & & \\
\hline fvo,k $(12 \%)$ & 1,15 & & \\
\hline T2 & $46,59 \mathrm{a}$ & $1,11 \mathrm{a}$ & $7.364,83 \mathrm{a}$ \\
\hline$(\mathrm{CV} \%)$ & 6,8 & 6,8 & 23,31 \\
\hline fm.k (12\%) & 46,84 & & \\
\hline fvo,k $(12 \%)$ & 1,12 & & \\
\hline T3 & $44,51 \mathrm{a}$ & $1,06 \mathrm{a}$ & $7.709,60 \mathrm{a}$ \\
\hline (CV\%) & 1,97 & 1,97 & 16,19 \\
\hline fm.k $(12 \%)$ & 49,48 & & \\
\hline fvo,k (12\%) & 1,18 & & \\
\hline
\end{tabular}

Fonte: Autores (2019)

Em que: Médias seguidas pela mesma letra na coluna não diferem estatisticamente pelo teste de Scott Knott com 5\% de significância. Em que: CV (\%) = Coeficiente de variação em porcentagem.; fm.k (12\%) = Resistência a tensão normal característica corrigida para $12 \%$ de umidade; fvo,k (12\%) = Resistência ao cisalhamento característico corrigido para $12 \%$ de umidade.

Desse modo, a utilização de lâminas com presença de nós de Cryptomeria japonica, classificadas conforme a ASTM D 245 (2006), não influencia na capacidade de carga de vigas de MLC. Ressalta-se que esse comportamento ocorreu em vigas com controle de qualidade feito pela distribuição sistemática das lâminas classificadas mecanicamente.

Ao comparar o menor valor característico da resistência axial obtido entre todos os tratamentos (46,84 MPa - T2), com o valor requisitado pela ABNT NBR 7190 (1997), observase que é atendido o valor mínimo de $20 \mathrm{MPa}$. Desse modo, pode-se utilizar os critérios de 
dimensionamento da classe C-20 para vigas de madeira de Cryptomeria japonica unidas por adesivo a base de poliuretano.

Esse resultado é alcançado devido ao efeito da homogeneização proveniente da classificação mecânica em todas as lâminas que compõem a MLC. Como a madeira sólida apresenta maiores variações das propriedades mecânicas na direção transversal (sentido medulacasca), ao classificar as lâminas pelo $\mathrm{E}_{\mathrm{w}}$ essas variações tornam-se menores. Desse modo, segundo Yang et al. (2007), a classificação mecânica e disposição das lâminas pode aumentar a resistência e rigidez do elemento MLC, uma vez que as propriedades do elemento passam a ser do compósito ao invés das propriedades individuais das lâminas.

Assim, o uso de lâminas com defeitos (tratamentos T2 e T3) podem ser utilizadas para compor vigas de MLC de Cryptomeria japonica, desde que haja uma classificação mecânica da rigidez para dispor as lâminas na viga corroborando com a literatura (BODIG; JAYNE, 1993; MIOTTO; DIAS, 2009; TEREZO; SZÜCS, 2010; ALMEIDA et al., 2011).

Os resultados médios da resistência ao cisalhamento e tração normal na linha de cola são mostrados nas Tabela 4.

Tabela 4 - Resistências ao cisalhamento paralelo e tração normal à linha de cola

Table 4 - Strengths to shear parallel and normal tensile to the glue line

\begin{tabular}{|c|c|c|c|c|c|c|}
\hline & \multicolumn{6}{|c|}{ Tensão de Ruptura (MPa) } \\
\hline & & Test. & T1 & T2 & T3 & p-valor \\
\hline \multirow{4}{*}{ Cisalhamento } & Média & $1,72 \mathrm{a}$ & $1,92 \mathrm{a}$ & $1,56 a$ & $1,74 \mathrm{a}$ & 0,1351 \\
\hline & D. P. & 0,42 & 0,37 & 0,24 & 0,39 & \\
\hline & CV (\%) & 24,09 & 19,38 & 15,44 & 22,29 & \\
\hline & fvo,k (12\%) & 1,11 & 1,64 & 1,13 & 1,61 & \\
\hline \multirow[t]{4}{*}{ Tração normal } & Média & $1,87 \mathrm{a}$ & $2,03 \mathrm{a}$ & $1,51 \mathrm{~b}$ & $1,71 \mathrm{a}$ & 0,0061 \\
\hline & D. P. & 0,44 & 0,46 & 0,35 & 0,18 & \\
\hline & CV (\%) & 23,34 & 22,72 & 23,22 & 10,43 & \\
\hline & fvo,k (12\%) & 1,42 & 1,38 & 1,45 & 1,28 & \\
\hline
\end{tabular}

Fonte: Autores (2019)

Em que: D.P. = Desvio padrão; CV (\%) = Coeficiente de variação em porcentagem; fvo,k (12\%) = Resistência ao cisalhamento característico a $12 \%$ de umidade.

Diferenças estatisticamente significativas não foram observadas entre os tratamentos para a resistência ao cisalhamento na linha de cola. No teste de resistência à tração normal, o tratamento T2 apresentou resistência inferior aos demais, contudo isso pode ter ocorrido devido a própria variabilidade da madeira, e não pelo uso das lâminas com defeitos; uma vez que o tratamento T3 também tem lâminas com defeitos classificados por S1, S2 e S3 na composição e teve resistência a tração normal estatisticamente igual aos tratamentos Testemunha e T1.

Ao comparar dentro dos tratamentos os valores de cisalhamento na linha de cola com o valor da ruptura por cisalhamento nas vigas, constatou-se que as mesmas não romperam por forças de cisalhamento, sendo que o valor médio de ruptura das vigas foi inferior ao da resistência ao cisalhamento na linha de cola. Desse modo, houve boa colagem das lâminas indicando que o processo de colagem foi eficiente. 


\section{Conclusão}

A madeira sólida de Cryptomeria japonica com 13 anos de idade não apresentou as propriedades mecânicas mínimas para ser classificada como estrutural pela NBR 7190 (ABNT, 1997). Entretanto, pela norma europeia EN 338 (2016) a espécie apresenta classificação C-16 para uso estrutural.

O uso da madeira de Cryptomeria japonica em vigas de MLC apresentou os valores mínimos necessários para uso estrutural, confirmando seu potencial para ser empregada no dimensionamento de estruturas pela NBR 7190 (ABNT, 1997) como classe C-20. Não foi constatada influência na diminuição da resistência das vigas MLC ao utilizar lâminas com defeitos. Isso possibilita o seu uso, desde que estejam dentro da classificação preconizada pela Norma e que haja também uma classificação mecânica da elasticidade para dispor as lâminas na viga.

\section{Referências}

ALMEIDA, D. H. et al. Caracterização completa da madeira da espécie amazônica Paricá (Schizolobium amazonicum Herb) em peças de dimensões estruturais. Revista Árvore, Viçosa, MG, v. 37, n. 6, p. 1175-1181, 2013. DOI: 10.1590/S0100-67622013000600019.

ALMEIDA, D. H. et al. Madeira Laminada Colada (MLC) da espécie Paricá. Revista da Madeira, Curitiba, v. 12, n. 30, p. 71-82, 2011.

AMERICAN SOCIETY OF TESTING AND MATERIALS.ASTMD 245: Standard Practice for Establishing Structural Grades and Related Allowable Properties for Visually Graded Lumber. West Conshohocken, 2006. 16 p.

AMERICAN SOCIETY OF TESTING AND MATERIALS.ASTMD 4761: Mechanical properties of Lumber and Wood-Base Structural Material. Philadelphia, 2013. 11 p.

ASSOCIAÇÃO BRASILEIRA DE NORMAS TÉCNICAS. NBR 7190: Projetos de estruturas de madeira. Rio de Janeiro, 1997. 107 p.

ASSOCIATION FRANCAISE DE NORMALISATION. NFB 5-32: Essai de cisaillement. Paris, 1942.

BODIG, J.; JAYNE, B. A. Mechanics of wood and wood composites. Nova York: Krieger Publishing, 1993. $712 \mathrm{p}$.

CARNEIRO, M. E.; BITENCOURT, E.; MUNIZ, G. I. B. Qualidade da madeira de Cryptomeriajaponica D. Don. Revista Floresta, Curitiba, v. 39, n. 4, p. 913-920, 2009. DOI: 10.5380/ RF. V39I4.16328.

CARPANEZZI, A. A. et al. Zoneamento ecológico para plantios florestais no Estado de Santa Catarina. Curitiba: EMBRAPA, 1988.

CARREIRA, M. R.; DIAS, A. A.; CALIL JUNIOR, C. Proposta de método para classificação visual estrutural de coníferas. Madeira, [s. l.], v. 5, n. 13, p. 23, 2004.

COMISIÓN PANAMERICANA DE NORMAS TÉCNICAS. COPANT 461: Método de 
determinación del peso específico aparente. Buenos Aires, 1972. 6 p.

COMISIÓN PANAMERICANA DE NORMAS TÉCNICAS. COPANT 464: Método de determinación dez la compresión axil o paralelo al grano. Buenos Aires, 1972. 4 p.

COMISIÓN PANAMERICANA DE NORMAS TÉCNICAS. COPANT 555: Método de Ensayo de Flexión Estática. Buenos Aires, 1973. 10 p.

CUNHA, A. B.; MATOS, J. L. M. Determinação do módulo de elasticidade em madeira laminada colada por meio de ensaio não destrutivo ("stress wave timer"). Revista Árvore, Viçosa, MG, v. 34, n. 2, p. 345-354, 2010. DOI: 10.1590/S0100-67622010000200018.

EUROPEAN STANDARD. EN 338: Structural timber - strength classes. Brussels, 2016. 16 p.

FONTE, A. P. N. et al. Propriedades físicas e químicas da madeira de cerne e alburno de Cryptomeria japonica. Revista de Ciências Agroveterinárias, Lages, v. 16, n. 3, p. 277-285, 2017. DOI: $10.5965 / 223811711632017277$.

IWAKIRI, S. et al. Avaliação da resistência de juntas coladas da madeira de Cryptomeria japonica e Sequoia sempervirens com diferentes adesivos. Scientia Forestalis, Piracicaba, v. 43, n. 105, p. 19-26, 2015.

LUKACEVIC, M.; FÜSSL, J. Numerical simulation tool for wooden boards with physically based approach to identify structural failure. European Journal of Wood and Wood Products, Berlin, v. 72, n. 4, p. 497-508, 2014.

MIOTTO, J. L.; DIAS, A. A. Produção e avaliação de vigas de madeira laminada colada confeccionadas com lâminas de eucalipto. Revista Tecnológica, Maringá, nesp, p. 35-45, 2009.

MOORE, J. R.; COWN, D. J. Corewood (juvenile wood) and its impact on wood utilization. Current Forestry Reports, [s. l.], v. 3, n. 2, p. 107-118, 2017. DOI: 10.1007/s40725-017-0055-2.

TEREZO, R. F.; SZÜCS, C. A. Análise de desempenho de vigas em madeira laminada colada de Paricá (Schizolobium Amazonicum Huber ex. Ducke). Scientia Forestalis, Piracicaba, v. 38, n. 87, p. 471-480, 2010.

VIDAURRE, G. G. et al. Lenho juvenil e adulto e as propriedades da madeira. Revista Floresta e Ambiente, Seropédica, v. 18, n. 4, p. 469-480, 2011. DOI: 10.4322/FLORAM.2011.066.

YANG, T-H. et al. Effect of laminate configuration on the modulus of elasticity of glulam evaluated using a strain gauge method. Journal of Wood Science, Singapura, v. 53, n. 1, p. 31-39, 2007. DOI: 10.1007/S10086-006-0818-Z. 